\title{
nature/methods
}

\section{Focus on next-generation sequencing data analysis}

\author{
A user's guide
}

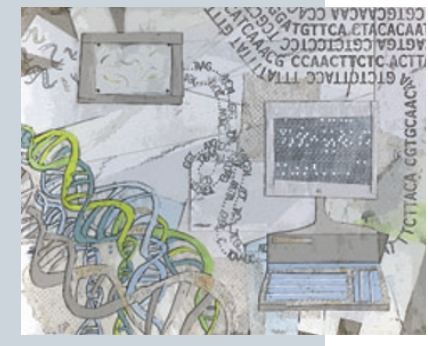

An artistic interpretation of the sequencing process from the DNA molecule to the decoded bases by Erin Dewalt.

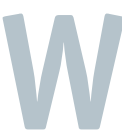

hat used to take years and extensive collaborationsgenerating the raw sequence of the three gigabases in the human genome-can now be done in a few days by a single investigator using a single run on some of the latest nextgeneration sequencing machines. The drawback is that this massive amount of data comes in the form of short reads, and one needs to invest heavily in computational analysis and choose from a plethora of tools to make sense of it all.

The recurring theme when it comes to the choice of software is that a 'one-size-fits-all' program does not exist, but users have to mix and match, which requires knowledge about the analysis steps in a given application and how different software operates at each step. This Focus aims to guide readers in their choice of software so they can extract a maximum of information from the data.

Once the sequence reads are generated, the elementary step common to all applications is to align them to a reference or, if no reference is available, to assemble them de novo. Our first review explains the principle behind current alignment and assembly methods. The second review describes the different signatures created by structural variants and software suitable to detect each signature. Some datasets have unique features, such as those derived from chromatin immunoprecipitation (ChIP) or cDNA libraries, as in ChIP-sequencing (ChIP-seq) or RNA-sequencing (RNA-seq) experiments, respectively. These datasets require a different set of software, as discussed in the third review.

We realize that for some of these applications new algorithms are still emerging at a rapid rate. The goal of our authors was to explain the principles behind existing programs and to take the readers through the different analysis steps of an application so that they can make informed choices about software suitable for their needs.

We are pleased to acknowledge the financial support of Applied Biosystems. As always, Nature Methods carries sole responsibility for all editorial content and peer review.

\section{Nicole Rusk}

PUBLISHED ONLINE 15 OCTOBER 2009; DOI:10.1038/NMETH.F.271

\section{CONTENTS}

S2 Next-generation gap

John D McPherson

S6 Sense from sequence reads: methods for alignment and assembly

Paul Flicek \& Ewan Birney S13 Computational
methods for

discovering structural variation with next-generation sequencing

Paul Medvedev,

Monica Stanciu \&

Michael Brudno

S22 Computation for ChIP-seq and RNA-seq studies

Shirley Pepke, Barbara Wold \& Ali Mortazavi

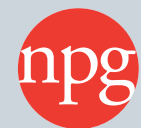

nature publishing group
Editor, Nature Methods Daniel Evanko Managing Production Editor

Focus Editor Nicole Rusk

Publisher Veronique Kiermer

Senior Copy Editors Anita Gould,

Irene Kaganman
Ingrid McNamara

Senior Production Editor

Brandy Cafarella

Production Editor Amanda Crawford
Design Erin Dewalt

Sponsorship Graham Combe

Marketing Joanna Budukiewicz 